\title{
Questes
}

Revue pluridisciplinaire d'études médiévales

Journée d'étude 1 - Trier, classer, organiser | 2022

Trier, classer, organiser

\section{La liste et la course : les listes bestournées du Roman de Renart}

Nicolas Garnier

\section{OpenEdition}

1 Journals

\section{Édition électronique}

URL : https://journals.openedition.org/questes/5997

DOI : 10.4000/questes.5997

ISSN : 2109-9472

Éditeur

Les Amis de Questes

Édition imprimée

Pagination : 141-155

ISSN : 2102-7188

Référence électronique

Nicolas Garnier, "La liste et la course : les listes bestournées du Roman de Renart », Questes [En ligne], Journée d'étude 1 - Trier, classer, organiser | 2022, mis en ligne le 31 janvier 2022, consulté le 21 février 2022. URL : http://journals.openedition.org/questes/5997 ; DOI : https://doi.org/10.4000/ questes.5997 


\title{
La liste et la course : les listes bestournées du Roman de Renart
}

\author{
Nicolas GARNIER
}

Sorbonne Université

«Mon nom est Légion, car nous sommes plusieurs »: on ne pourrait mieux définir l'aspect protéiforme du Roman de Renart, que par cette citation de l'Évangile de Marc'1. Les récits renardiens sont définis par une propension au développement: on ne compte plus les rebondissements, les digressions, les amplifications qui accroissent la narration. L'énumération, qu'elle soit le fait de Renart ou des narrateurs, trouve donc toute sa place dans le Roman: on se souvient des nombreuses confessions du personnage éponyme, que ce soit dans la branche nommée à juste titre Confessions Renart, ou dans celle du Siège de Maupertuis, où le goupil énumère tous ses sévices avec gourmandise.

Ces boursouflures langagières sont à l'origine de nombreuses listes au sein des différentes branches du Roman. Madeleine Jeay, dans son travail sur la liste dans la littérature médiévale, a consacré quelques pages à cette question, en particulier sur les listes littéraires de l'œuvre, c'est-àdire sur ces moments où le Roman cite abondamment d'autres œuvres et d'autres genres médiévaux ${ }^{2}$. Les récits renardiens peuvent être considérés comme l'un des types de textes médiévaux les plus porteurs de listes avec

\footnotetext{
1 Jean Scheidegger utilisait pour sa part cette citation biblique pour définir la multiplicité des auteurs du Roman de Renart (Le Roman de Renart ou le texte de la dérision, Genève, Droz, 1989, p. 94).

${ }^{2}$ Madeleine Jeay, Le Commerce des mots. L'usage des listes dans la littérature médiévale (XII ${ }^{e}-\mathrm{XV}^{e}$ siècles), Genève, Droz, 2006.
} 
les chansons de geste. Certaines listes dans le Roman de Renart ont souvent été associées aux listes épiques : ce sont celles qui répertorient un ensemble d'animaux ou d'hommes. On ne recense plus les catalogues de vassaux dans la chanson de geste, dénombrant les combattants ${ }^{3}$. Dans le Roman de Renart, en l'occurrence, il s'agit principalement de scènes de poursuite : ainsi, dans le Jugement de Renart, des humains traquent l'ours Brun, tandis que dans la branche l'Escondit, c'est le goupil qui est pourchassé par une meute de chiens. À chaque fois, le récit nous propose une pause dans la narration qui dresse une liste des assaillants, ce qui correspond bien à la définition proposée par Madeleine Jeay, pour qui «l'essentiel est précisément l'effet de rupture, de présence dans l'énoncé hôte d'un bloc textuel identifiable par son hétérogénéité ${ }^{4}$. Or ces listes, présentes dans des branches différentes, ont souvent attiré l'attention de la critique qui y a vu justement un lien avec l'épopée, sur le mode parodique. Ainsi, dès 1914, Lucien Foulet affirmait : «Mais lisez cette énumération de noms retentissants [...], et vous croirez voir toute une armée de traîtres Sarrasins acharnée à barrer la route à Guillaume qui fuit vers Orange ${ }^{5} \gg$.

Néanmoins, s'il est vrai que ces effets d'énumération d'animaux ou d'humains permettent toujours une hyperbole épique se produisant lors de folles poursuites, certains aspects de ces listes ne peuvent être réduits à une parodie. On constate que si ces énumérations se retrouvent dans de nombreux récits renardiens, les deux branches qui sont les plus riches pour cet effet sont celles du Jugement de Renart et de l'Escondit. On exclura de fait de notre étude les listes, bien que nombreuses, de la branche Renart empereur. En effet, ce récit, où les troupes du roi Noble

\footnotetext{
${ }^{3}$ Ibid., p. 46

${ }^{4}$ Ibid., p. 9.

${ }^{5}$ Lucien Foulet, Le Roman de Renart, Paris, Honoré Champion, 1914, p. 215.

L'auteur fait ici référence aux sarrasins poursuivant Guillaume dans Aliscans.
} 
affrontent les païens dirigés par un chameau, avant de retourner guerroyer contre Renart le traître, est bien trop ancré dans le tropisme humain au détriment de l'animal, et reprend beaucoup plus explicitement les modèles des chansons de geste et celui de la guerre entre Arthur et Mordred $^{6}$. Il s'agit donc de la liste des combattants allant s'affronter, et non de celle des animaux qui en poursuivent un autre, comme c'est le cas dans les extraits qui nous intéressent. Qui plus est, dans le Jugement et l'Escondit, on voit systématiquement s'opposer une liste animale à une liste humaine au sein de la même branche, ce qui met davantage au premier plan la question de l'humanité et de l'animalité, constitutive du roman. Enfin, reléguer ces listes à une simple parodie épique empêche de s'interroger véritablement sur le fonctionnement poétique de ces catalogues. En effet, d'un manuscrit à l'autre, les listes ne sont pas toujours totalement identiques, notamment sur leurs choix onomastiques. Il s'agira donc d'analyser ces listes en elles-mêmes pour en dévoiler tous les ressorts rhétoriques, mais également de voir comment elles s'insèrent dans le récit, et ce qu'elles nous permettent de comprendre du Roman de Renart.

\section{Les listes renardiennes : une parodie épique?}

L'énumération des combattants dans la chanson de geste est un motif qui peut se produire avant un combat ou, dans le cas qui nous intéresse, lorsque des guerriers en poursuivent un autre : c'est ce dernier cas qu'on retrouve essentiellement dans le Roman de Renart. La liste du Jugement fait exception, puisqu'elle énumère les plaignants s'acharnant sur le goupil: mais ne s'agit-il pas là après tout d'une poursuite

\footnotetext{
${ }^{6}$ Voir Roger Bellon, " "Renart empereur". Le Roman de Renart, ms. H, branche XVI : une réécriture renardienne de La mort le roi Artu? ", Cahiers de recherches médiévales, 15, 2008, p. 3-17
} 
judiciaire? La plus connue de ces énumérations, car la plus emblématique, est celle qui se situe à la fin de la branche dite de l'Escondit, où Renart, flairant un piège, préfère prendre ses pattes à son cou, et se faire alors poursuivre par une meute de chiens, tous nommés. C'est d'ailleurs la plus longue de celle que nous proposons : soixantedouze vers dans l'édition Martin énumèrent les noms des différents chiens courant après Renart ${ }^{7}$. La référence à l'épopée se fait d'entrée de jeu, comme le remarque Roger Bellon : «Pour ouvrir cet épisode, dont la teneur parodique ne fait aucun doute et comme pour signer son intention de se livrer à la parodie d'un motif épique, l'auteur arrête son récit et en appelle à l'intention du lecteur en utilisant une formule traditionnelle des poèmes épiques "Ja m'orrez dire qui il furent ${ }^{8}$." " De façon générale, si on ne trouve pas ce genre d'apostrophes dans toutes les listes, on peut remarquer la multiplication des verbes au présent, qui permet à la fois de renforcer l'aspect visuel du passage, tout en marquant la rupture avec la narration pure.

C'est surtout l'onomastique qui caractérise ces listes et qui les lie à leur référent épique. Dans l'épopée et dans le Roman de Renart, on observe des similitudes concernant l'aspect sonore et sémantique de ces noms. Philippe Ménard a montré comment les noms dans les chansons de geste, principalement ceux des Sarrasins, étaient très significatifs, avec des initiales et finales du nom qui suggèrent l'exotisme, comme les finales en «is » pour Corsalis, Margaris ou en «ot» pour Astrigatot, Bagot... Quant aux préfixes, ils sont souvent porteurs de sens, comme

\footnotetext{
${ }^{7}$ Le Roman de Renart, éd. et trad. Jean Dufournet et Andrée Méline, Paris, Flammarion, coll. GF, 1985, t. I, p. 394-398, v. 185-1257 (cette édition de poche reprend l'édition Martin, Strasbourg, Trübner, 1882-1887).

${ }^{8}$ Roger Bellon, «La parodie épique dans les premières branches du Roman de Renart », dans Épopée animale, fable, fabliau : actes du IV colloque de la Société Internationale Renardienne, dir. Gabriel Bianciotto et Michel Salvat, Paris, PUF, 1984, p. 81.
} 
ceux en «fal » qui annoncent la fausseté, évoquant le substantif « félon » avec Falsaron, Faldrun ${ }^{9}$... On remarque que ces noms allient deux procédés : celui du jeu sonore pur, où seules comptent l'assonance ou l'allitération exotisantes, et le nom richement sémantisé, où le patronyme reflète la personne qui le porte. Or, selon Roger Bellon, on retrouve ce procédé dans la liste des chiens qui poursuivent Renart, où on a bien là aussi des noms chargés de senefiance, dont le sens définit le porteur, forgés à partir d'un verbe ou d'un substantif tels que Espinars ou Hurtevilein, mais aussi des noms qui ne valent que pour leur sonorité expressive, comme dans les duos Ferin et Frias $^{10}$. On retrouve cela dans les autres listes: si on reprend les paysans s'attaquant à Brun dans le Jugement, on a bien des noms sémantiquement forts comme «Hardoïn Copevilein ${ }^{11} \gg$. La différence notable est que les noms n'évoquent pas un ailleurs lointain, mais sont des noms typiques de paysans, comme Gonbertz, Hubert, Martin... Dans tous les cas, on retrouve dans ces listes des patronymes directement issus des chansons de geste : ainsi la liste des chiens évoque-t-elle tout aussi bien héros chrétiens comme Olivier, que sarrasins, tel Amirant, quand les paysans se prénomment Ogier ou Otran. Ces listes renardiennes reprennent bien des procédés de la chanson de geste, voire empruntent plus directement leurs patronymes.

Mais la simple reprise ne suffit pas pour qu'il y ait parodie. Pour Roger Bellon, c'est le cadre spécifique de l'animalité dans le Roman de Renart qui permet de se livrer à la parodie des chansons de geste. L'ensemble du roman semble basé sur cette interférence des mondes humain et animal qui se développerait surtout dans deux motifs du

\footnotetext{
${ }^{9}$ Philippe Ménard, Le Rire et le sourire dans le roman courtois en France au Moyen Âge, Genève, Droz, 1969, p. 46-47

${ }^{10}$ Roger Bellon, «La Parodie épique dans les premières branches du Roman de Renart », art. cit., p. 84.

${ }^{11}$ Le Roman de Renart, éd. cit., p. 74, v. 656.
} 
roman, issus de la tradition épique : le songe prémonitoire et la poursuite de l'ennemi en fuite. Ainsi, l'utilisation d'animaux, en l'occurrence de chiens dans l'exemple développé par Roger Bellon, viendrait rabaisser la forme épique du passage. Certains noms sont déjà porteurs de comique, comme Escoillez, mais de façon générale, le rappel persistant au monde animal conduirait à rabaisser le contexte épique : «Le procédé le plus utilisé est celui que nous pourrions appeler l'encadrement : une formule, une expression, un procédé empruntés à l'épopée (l'emprunt intégrant alors le récit dans l'univers épique) sont entourés de termes désintégrateurs qui nous rappellent que les personnages sont en premier lieu des animaux et le restent sous l'habillage humain que leur donne le conteur $^{12} »$. Toutefois, on peut remarquer que ce rabaissement héroïcomique des listes ne fonctionne pas uniquement grâce au versant animalier du Roman. Ainsi, la liste évoquée plus haut des poursuivants de Brun reprend également des éléments épiques, mais ici le rabaissement ne se fait pas par le biais animal, mais par celui des paysans. On retrouve également des patronymes au potentiel comique non négligeable, comme Hubert Grospet. L'animalité n'est donc pas le seul versant utilisé pour cette réappropriation décalée de l'épopée médiévale ${ }^{13}$.

De surcroît, Philippe Ménard remarquait déjà que les énumérations des chansons de geste «ne manqu[ai]ent pas de saveur ${ }^{14} »$. Il existe donc un jeu onomastique présent dans les chansons de geste, qui ne paraît être qu'accentué par le Roman de Renart, renforcé par un aspect trivial, qu'il soit animal ou paysan. La liste n'est-elle pas déjà le lieu de toutes les

\footnotetext{
${ }^{12}$ Roger Bellon, «La Parodie épique dans les premières branches du Roman de Renart », art. cit., p. 84.

${ }^{13}$ Sur ces questions, voire Armand Strubel, «Ironie, parodie, allégorie : le rire "renardien" et la branche I », Méthode!, 13, 2007, p. 21-31 et Dominique Boutet, Poétiques médiévales de l'entre-deux, ou le désir d'ambiguïté, Paris, Honoré Champion, coll. Essais sur le Moyen Âge, 2017, notamment p. 159-171.

${ }^{14}$ Philippe Ménard, Le Rire et le sourire, op. cit., p. 12.
} 
fantaisies verbales, fantaisie qu'on retrouve par exemple dans les listes des chevaliers de la Table Ronde dans Érec et Énide selon Danièle James-Raoul $^{15}$ ? Or la fantaisie verbale caractérise aussi bien le récit que le héros éponyme du Roman de Renart ${ }^{16}$. Dès lors, comment le roman pouvait-il résister à la tentation d'un effet de style qui se prête à merveille à toutes les extravagances langagières ?

\section{Versions et variantes : le plaisir de la liste}

Ce plaisir du langage foisonnant semble se manifester particulièrement dans la diversité des manuscrits qui nous livrent le Roman de Renart. Lucien Foulet rappelait ainsi qu' « il n’y pas un roman de Renart, il y en a vingt-huit ${ }^{17} \gg$. On trouve de fait quatorze manuscrits qui dévoilent chacun différentes branches, dans un ordre souvent variable, sans compter les seize manuscrits fragmentaires ou partiels. L'ensemble de ces versions a été classé en différentes familles par les critiques modernes ${ }^{18}$. Or, d'une famille de manuscrit à l'autre, les textes peuvent connaître des variantes assez importantes, qui dépassent un simple agencement de branches divergentes. C'est principalement le cas pour la branche de l'Escondit: dans l'édition Martin, où elle porte le numéro Va, elle voit Renart se faire piéger par Ysengrin et le chien

15 Danièle James-Raoul, Chrétien de Troyes, la griffe d'un style, Paris, Honoré Champion, 2007, p. 326-334.

${ }^{16}$ Claude Reichler parle même de «fête du langage » (La Diabolie, la séduction, la renardie, l'écriture, Paris, Éditions de Minuit, 1979, p. 104).

${ }^{17}$ Lucien Foulet, Le Roman de Renart, op. cit., p. 565.

${ }^{18}$ Pour une analyse plus poussée de cette foisonnante tradition manuscrite du Roman de Renart, voir les travaux d'Ernest Martin, Examen critique des manuscrits $d u$ Roman de Renart, Bâle, Schweighauser, 1872 et ceux de Herman Büttner, Studien zu den Roman de Renart un den Reinhart Fuchs, Die Überlieferung des Roman de Renart und die Handschrift o, Strasbourg, Trübner, 1891. Kenneth Varty a remis en cause le classement traditionnel des manuscrits dans son article « The transformations of Pierre de St Cloud's Roman de Renart within the Renart manuscripts », dans Farai chansoneta novele. Hommage à Jean-Charles Payen, Caen, Centre de Publication de l'Université, 1989, p. 419-435. 
Roonel. Il doit alors s'enfuir devant une meute de chiens. Dans l'édition de Mario Roques où elle est la branche VII, c'est Renart qui parvient à piéger la cour, en ramenant une troupe de paysans parmi les animaux après avoir une nouvelle fois dupé Brun et Tybert ${ }^{19}$. On voit ici tout l'intérêt de comparer les deux familles de manuscrits, puisque cela aboutit à deux listes différentes, une de chiens pourchassant Renart et une de paysans attaquant la cour de Noble. Dans un cas, Renart est perdant, dans l'autre, il triomphe.

Dans l'édition Mario Roques, il faudrait plutôt parler de deux listes d'hommes. En effet, on a d'abord les humains qui s'en prennent à l'ours et au chat coincés dans leur maison (v. 1515-1524) et le retour de ces paysans lorsqu'ils s'attaquent à la cour animalière (v. 1677-1688). On retrouve d'ailleurs certains noms dans ces inventaires humains, comme Tigier Brisefouace, aux vers 1515 et 1685. Globalement néanmoins, les noms sont différents entre les deux listes, même si on peut dégager une homophonie, avec de nombreuses finales en -ert, sans que cela soit forcément dû à la rime, comme Forbert, Gombert, Houdebert, Robert... À chaque fois s'ensuit une scène de bataille héroï-comique où les paysans s'en prennent aux animaux; on reste donc dans une tonalité qui se veut épique.

Il est difficile en tout cas de comparer la liste d'humains de l'édition Roques avec celle de chiens de l'édition Martin. Par contre, les listes de ces deux éditions présentent des similitudes avec celles de la famille $\gamma$, éditée par Naoyuki Fukumoto, Noboru Harano et Satoru Suzuki ${ }^{20}$. En effet, $\gamma$ est souvent considéré comme une fusion de $\alpha$ et $\beta^{21}$.

\footnotetext{
${ }^{19}$ Le Roman de Renart édité d'après le manuscrit de Cangé, éd. Mario Roques, Paris, Champion, coll. Classiques Français du Moyen Âge, t. III, 1973.

${ }^{20}$ Le Roman de Renart, éd. Naoyuki Fukumoto, Noboru Harano et Satoru Suzuki, Tokyo, France-Tosho, t. I,1983. Par commodité, on parlera de cette édition comme l'édition F-H-S.
} 
Or c'est exactement ce qu'on retrouve dans l'Escondit, puisqu'on a la liste des paysans attaquant les animaux, comme dans l'édition Roques, avant que Renart ne retourne à la cour et ne soit poursuivi par les chiens, comme dans l'édition Martin, créant donc une branche à l'aspect déséquilibré et très hétérogène. Dès lors, on retrouve quasiment à l'identique les mêmes listes entre les deux familles, à quelques variantes près. Ainsi, dans l'édition Roques, Fremeris et Voide Escuele ${ }^{22}$ sont deux personnages réunis en un seul dans l'édition $\mathrm{F}-\mathrm{H}-\mathrm{S}$, répondant au nom de Ferneris Vide-escuele au vers $1166^{23}$. F-H-S reprend également les mêmes formules rhétoriques d'ouverture que la liste des chiens de l'édition Martin, et les variantes patronymiques sont très réduites, comme les chiens Roilliez, Loviaus et Amiranz ${ }^{24}$, nommés Foillet, Lovel et Amirant dans l'édition Martin ${ }^{25}$. Les changements sont donc minimes et très proches d'un point de vue sonore. Les listes font d'ailleurs soixantedeux vers dans les deux cas. Par contre, pour les manuscrits composites $\mathrm{O}$ et $\mathrm{H}^{26}$, dont le récit de l'Escondit est proche de Martin, on remarque avec Aurélie Barre concernant le premier que « dans cette branche, le copiste de $\mathrm{O}$ ajoute 6 vers originaux amplifiant la liste des chiens lancés à la poursuite de Renart. Ce sont les vers 1259 à 1262 et $1266-1267^{27} \gg$. Le seul changement produit est davantage une amplification qu'une modification en profondeur. À l'inverse, le manuscrit $\mathrm{H}$ résume

\footnotetext{
${ }^{21}$ Jean Scheidegger, Le Roman de Renart ou le texte de la dérision, op. cit., p. 25.

${ }^{22}$ Le Roman de Renart, éd. cit. (Mario Roques), p. 52, v. 7234.

${ }^{23}$ Le Roman de Renart, éd. cit., (F-H-S), p. 176.

${ }^{24}$ Ibid., p. 179, v. 1254.

${ }^{25}$ Le Roman de Renart, éd. cit. (Dufournet), p. 396, v. 1198.

${ }^{26}$ Pour une présentation des différents manuscrits, voir la préface d'Ernest Martin à son édition du Roman de Renart (p. I-XXVII).

${ }^{27}$ Le Roman de Renart, éd. Aurélie Barre, Berlin/New York, De Gruyter, 2010, p. 40, note 53.
} 
grandement le texte: la liste des chiens ne fait que dix-neuf vers, à comparer avec les soixante-deux vers de l'édition Martin ${ }^{28}$.

Contrairement à ce qu'on a pu constater pour la branche l'Escondit, la branche du Jugement de Renart ne connaît pas de variante significative du point de vue du récit, mais on remarque des changements plus importants dans ses listes. Ainsi, les noms des paysans se lançant à l'attaque de l'ours Brun sont très différents d'une édition à l'autre : on en retrouve certains, comme Tigier, mais appelé «li forniers de la ville ${ }^{29}$ » chez Martin, et surnommé « de la Place ${ }^{30} »$ chez Roques. Comme dans l'Escondit, on retrouve également des noms sémantiquement forts, à défaut d'être subtils, comme Joudö̈n Trouseputain dans l'édition Roques $^{31}$, mais aussi parfois un vers de développement sur tel ou tel personnage: ainsi, il nous est dit de Tigiers qu'il « esposa noire Cornille » dans l'édition Martin ${ }^{32}$. Une nuance intéressante apparaît aussi dans l'édition F-H-S : l'édition Martin parle ainsi d'un «Otrans li quens de l'Anglee / qui sa feme avoit etranglee ${ }^{33} »$ quand l'édition F-H-S parle de «Outrant, li cous de l'Aglee, / qui sa fame avoit estranglee ${ }^{34} »$. Le personnage passe ainsi du rang de comte à celui de cocu. La variante de l'édition F-H-S est intéressante, car elle semble expliquer le vers suivant, le rendant au passage plus comique et le rapproche du genre des fabliaux. Surtout, elle ravale le personnage socialement en lui ôtant son statut de comte. Toujours est-il que ces différentes listes montrent bien toute leur potentialité narrative. C'est en effet l'instant narratif qui permet le plus de

\footnotetext{
${ }^{28}$ Le Roman de Renart, éd. Armand Strubel, avec la collaboration de Roger Bellon, Dominique Boutet et Sylvie Lefèvre, Paris, Gallimard, coll. Bibliothèque de la Pléiade, 1998, p. 216-217, v. 1912-1931.

${ }^{29}$ Le Roman de Renart, éd. cit. (Dufournet), p. 74, v. 661.

${ }^{30}$ Le Roman de Renart, éd. cit. (Mario Roques), t. I, 1982, p. 23, v. 668.

${ }^{31}$ Ibid., p. 23, v. 657.

${ }^{32}$ Le Roman de Renart, éd. cit. (Dufournet), p. 76, v. 662.

${ }^{33}$ Ibid., p. 74, v. 659-660.

${ }^{34}$ Le Roman de Renart, éd. cit., (F-H-S), p. 205, v. 659-660.
} 
liberté à l'auteur et/ou au copiste : comme le rappelle Roger Bellon, ces listes peuvent parfaitement être supprimées du récit sans que la compréhension de ce dernier soit changée ${ }^{35}$. Dès lors, que le personnage se prénomme Robert ou Gombert ne change rien, et le narrateur peut s'amuser autant qu'il le veut, que ce soit d'un point de vue sonore en multipliant les assonances et allitérations, par la grivoiserie des patronymes ou encore par les très brèves biographies évoquées. On voit bien que nos deux branches étudiées proposent deux cas de figure : soit, comme dans le Jugement de Renart, la liste est le lieu de toutes les libertés poétiques, soit, comme dans l'Escondit, la liste ne compte pas véritablement, et la variance se fait véritablement d'un point de vue narratif, c'est-à-dire sur le moment du récit où intervient l'énumération, les seuls changements se faisant sur le nombre de vers d'un manuscrit à l'autre.

\section{La liste, entre l'homme et la bête}

Entre les deux branches que nous avons évoquées, on peut remarquer de nombreuses similitudes dans l'emploi de la liste. En effet, dans un cas comme dans l'autre, elles lient fortement monde humain et monde animal, puisqu'il n'y a pas de liste animale sans liste humaine : dans le Jugement de Renart, l'énumération des poursuivants humains de Brun est suivie par celles des animaux de la cour se plaignant de Renart puis le poursuivant une fois qu'il a trahi son serment de partir en croisade. Dans l'Escondit, on a bien vu qu'une liste de chiens avait été remplacée par deux listes humaines. On y retrouve également des listes d'animaux présents à la cour. À chaque fois, les mêmes procédés sont présents: noms porteurs de sens, souvent comiques, références

\footnotetext{
${ }^{35}$ Roger Bellon, «La parodie épique dans les premières branches du Roman de Renart», art. cit., p. 80.
} 
intertextuelles à l'épopée plus ou moins marquées... Ces deux types d'actants favorisent un registre bas: mais n'est-ce pas également un moyen de montrer la similitude entre ces deux univers ? Le monde paysan ne vaudrait alors pas mieux que le monde animal, et c'est d'ailleurs une idée fréquente du Roman, puisque Renart et ses comparses triomphent le plus souvent des hommes, maladroits et incultes ${ }^{36}$. Mais ne peut-on pas élargir le propos et montrer l'extrême porosité entre l'humain et l'animal, tous deux unis dans le rôle du pourchassant ? Jean Scheidegger comme Claude Reichler ont montré l'importance du désir au cœur du roman ${ }^{37}$. Or, justement, cette surenchère épique de poursuivants n'expose-t-elle pas l'importance de cette notion dans le récit? Tout le Roman semble traversé par cette thématique, entre poursuite de nourriture, poursuite de vengeance et poursuite judiciaire. La liste épique de la poursuite, par son ampleur hyperbolique, n'est là que pour rappeler ce point commun des êtres vivants, avant tout mus par cette pulsion.

Un autre point vient nous rappeler ce lien entre animalité et humanité qui se fait grâce à ces listes de la fuite : c'est la possible évocation des Métamorphoses d'Ovide, comme l'avait repéré Madeleine Jeay, et plus précisément l'épisode d'Actéon dans le livre III aux vers 206-233 ${ }^{38}$. En effet, dans ce passage, une fois Actéon transformé en cerf pour avoir aperçu la déesse Diane nue au bain, celui-ci est poursuivi par la meute de ses chiens de chasse, meute dont Ovide nous donne une liste de trente et un noms. Une fois encore, on retrouve donc une énumération de poursuivants, avec précision de leur nom et pour certains

\footnotetext{
${ }^{36}$ Voir Micheline Combarieu du Grès, «Les rapports entre les animaux et les hommes dans le Roman de Renart : langage de force et force du langage », dans À la recherche $d u$ "Roman de Renart», dir. Kenneth Varty, New Alyth, Lochee Publications, 1988-1991, tome I, p. 163-215.

${ }^{37}$ Jean Scheidegger, Le Roman de Renart ou le texte de la dérision, op. cit. ; Claude Reichler, La Diabolie, la séduction, la renardie, l'écriture, op. cit.

${ }^{38}$ Madeleine Jeay, Le Commerce des mots, op. cit., p. 171, $\mathrm{n}^{\mathrm{o}} 63$.
} 
de leurs traits spécifiques. Leurs noms aussi sont significatifs, car ils peuvent évoquer en grec les particularités de leur pelage par exemple. On voit donc bien le lien qu'on peut faire avec les chiens poursuivant Renart à la fin de l'Escondit. Faut-il une nouvelle fois y voir une intention parodique? Toujours est-il qu'on saisit parfaitement le lien entre humanité et animalité, puisque Actéon est un homme transformé en cerf. On peut suivre ici Dominique Boutet, pour qui le lien entre ces deux univers, humain et animal, dans le Roman de Renart, trahirait le rapport étroit qu'entretient ce dernier avec l'épopée dans sa dimension mythique, puisque les mythes lient fortement épique et animalité ${ }^{39}$. C'est le cas dans la mythologie antique comme ici avec l'exemple d'Actéon, ou encore dans la mythologie scandinave: on ne recense plus les métamorphoses animales ou les divinités zoomorphes qui parsèment ces récits. L'utilisation de la liste, par le biais de la référence à la fois à la chanson de geste et au mythe, semble bien réactualiser ce lien entre animalité et épopée. Ces listes peuvent donc bien être définies comme « bestournées »: elles sont tout à la fois détournées, et renvoyées à leur nature profondément animale.

Néanmoins, l'utilisation de poursuivants semble respecter un schéma assez strict selon la nature du fuyard : lorsque c'est Renart qui s'enfuit, les poursuivants sont toujours des animaux. En revanche, lorsque c'est un ennemi du goupil qui doit prendre ses pattes à son cou, les poursuivants sont des êtres humains. On peut ainsi remarquer que dans les deux systèmes, la liste permet de montrer l'isolement du personnage au milieu d'une énumération considérable. Toutefois, dans le premier cas, elle fait de Renart un personnage unique, aux prises avec l'ensemble du monde animal, seul contre tous, et donc capable de se

${ }^{39}$ Dominique Boutet, « Le Roman de Renart est-il une épopée ? », Romania, 126/3-4, 2008, p. 463-479, ici p. 475. 
sortir de toutes les situations ; dans l'autre cas, lorsque c'est l'adversaire $\mathrm{du}$ goupil qui doit affronter des vagues d'ennemis, cela montre au contraire la toute-puissance de Renart. En effet, c'est toujours à cause d'une ruse renardienne qu'un animal se voit poursuivi par des êtres humains: s'en prendre au goupil, c'est prendre le risque des pires représailles, et cette liste d'ennemis prend une tournure quasi tragique pour l'antagoniste de Renart. On peut également remarquer que les fuyards ne sont jamais des hommes: le personnage en fuite étant singularisé, cela tend bien à montrer que le Roman de Renart est bien avant tout un récit où les personnages principaux sont des animaux, et où les hommes ne sont que secondaires et noyés dans une foule.

L'Escondit et le Jugement suivent donc un schéma analogique. Il est d'ailleurs remarquable qu'il s'agisse des deux branches qui suivent à ce point ce procédé de la liste. Elles ne sont pourtant pas les seules à proposer un épisode d'un personnage renardien en fuite : ainsi, dans le Vilain Berthaud, Renart, après avoir lâché le coq Noiret, est poursuivi par des chiens, mais le texte ne mentionne que « quatre levrier [qui] viennent bruiant $t^{40} \gg$. Les longues listes sont donc le propre de nos deux branches, si l'on exclut les listes de guerriers de Renart Empereur. Or, il est intéressant de constater que ces deux branches présentent plus généralement de nombreuses similitudes, puisque ce sont deux récits essentiellement judiciaires, qui commencent par la plainte d'Ysengrin auprès de la cour du roi Noble. Ce procédé commun de la liste développée aux consonances épiques véritablement propre au Jugement et à l'Escondit permet de renforcer l'affirmation qu'il existe un principe de construction, du moins de structure, similaire entre les deux récits. Surtout, elle semble bien poser le lien entre poursuite physique et poursuite judiciaire.

\footnotetext{
${ }^{40}$ Le Roman de Renart, éd. cit. (Dufournet), t. II, p. 406, v. 640.
} 
Peut-on considérer les listes de poursuivants du Roman de Renart comme une parodie épique ? Certes, la référence est indéniable, ne seraitce que par cet effet d'accumulation hyperbolique. On ne saurait pourtant la réduire à ce simple registre. Les branches renardiennes semblent plutôt développer la fantaisie verbale déjà présente dans le procédé de l'énumération épique, fantaisie que traduisent parfaitement les variantes de manuscrits. On peut définitivement parler de listes épiques bestournées, puisqu'elles parviennent à la fois à subvertir un modèle, tout en montrant le rapport étroit que celui-ci entretient avec l'animalité. Les listes de poursuivants révèlent donc bien l'ensemble des enjeux du Roman, que ce soit le rapport entre humanité et animalité, l'aspect fondamental du désir, mais aussi la réécriture. 